\title{
The Mellin Transform and Euler-Maclaurin Summation Rule Applied to System of $N$ - Independent Harmonic Oscillators
}

\section{A Transformada de Mellin e o Método de Soma de Euler-Maclaurin Aplicados a um Sitema de $N$ Osciladores Harmônicos Independentes}

\author{
Antonio Edson Gonçalves ${ }^{1}$; Veríssimo Manoel de Aquino ${ }^{2}$
}

\begin{abstract}
We confront the Mellin and the Euler-Maclaurin summation rule when used in order to calculate $N$ independent harmonic oscillators thermodynamic potentials, and compare the approximate expressions with the exact ones for these quantities. The goal of this work is at least twofold: First, to compare the results obtained for the thermodynamic potentials of a system of $\mathrm{N}$ oscillators using the Mellin and the Euler-Maclaurin summation rule in order to have a discernment on which one is most appropriate for application to more complex systems with finite and infinite number of components. Second, to present to the reader the ideas of these techniques in a pedestrian way with the aim of providing one material with detailed calculation that can be useful like a basic reference to more deep calculation, in particular string theory and supergravity.
\end{abstract}

Keywords: Partition Function. Harmonic Oscillators. Thermodynamic Potentials. Euler-Maclaurin Formula. Mellin Transform.

\section{Resumo}

Neste trabalho apresentamos as expressões obtidas para os potenciais termodinâmicos de um sistema de $N$ osciladores independentes calculadas usando dois diferentes métodos. Expressões em forma de séries para os potenciais são obtidas usando a transformada de Mellin, e, alternativamente, usando a regra da soma de Euler-Maclaurin. A concordância dos resultados no limite de altas temperaturas é verificada. $\mathrm{O}$ trabalho tem dois objetivos. Primeiro, comparar os resultados obtidos pelos dois caminhos de forma a verificar qual o mais adequado para aplicação em casos mais complexos, por exemplo, para o estudo do comportamento dos potenciais termodinâmicos a partir de funções partições sugeridas para buracos negros ou em modelos de supergravidade. Segundo, apresentar ao leitor de forma simples e didática, as ferramentas básicas para o cálculo dos potenciais termodinâmicos, preparando-o para aplicação em outros sistemas. Neste sentido cálculos explícitos são desenvolvidos no decorrer do trabalho. Argumentos em favor da utilização da transformada de Mellin são apresentados.

Palavras-chave: Função Partição. Osciladores harmônicos. Potenciais Termodinâmicos. Fórmula de EulerMaclaurin. Transformada de Mellin.

\footnotetext{
${ }^{1}$ Dr. Prof. Depto. Física, UEL, Londrina, Pr, Brasil; E-mail: goncalve@uel.br

${ }^{2}$ Dr. Prof. Depto. Física, UEL, Londrina, Pr, Brasil; E-mail: verissim@uel.br
} 


\section{Introduction}

In the last times there was a great interest in the calculation of densities of states for some physical system, mainly black holes (STROMINGER; VAFA, 1996), thermodynamic properties of systems in the presence of gravitational field and thermodynamic properties in supergravity theories (WITTEN, 1988; WITTEN, 1989; WITTEN, 1990; WITTEN, 2001; WITTEN, 2002; WITTEN, 2007). The reason for that is the directly relation with gravitational quantization in quantum field theories in curved spacetime or string theory (POLCHINSKI, 1998a; POLCHINSKI, 1998b). This challenge of the contemporary physics is not new; this have directly relation with number theory in mathematics and appear in physics in the Bohr and Kalckar calculations about the densities of states of heavy atom nuclei (BOHR; KALCKAR, 1937), and Van Lier e Uhlenbeck who consider the calculation of density of energy levels of a nucleus by using the methods of Darwin-Fowler (saddle point). Actually in many papers the authors considers many different kinds of partitions functions, in the physical not mathematical sense, in order to understand, through approximative calculation or symmetries considerations, the thermodynamic properties of the physical system associated with these partitions functions (BOHR; KALCKAR, 1937). As a first steps in the calculation of the density of states or the entropy is the calculation of the thermodynamic potentials, that for the more complex systems can not be get exactly, but only through approximative methods. In this paper, for a system of $N$ independent harmonic oscillators, approximate values of thermodynamic potentials in the Mellin transform (GRADSHTEYN, 1984) approach and using the Euler-Maclaurin summation rule (ABRAMOWITZ; STEGUN, 1964) are carried out in order to detect the most adequate to applications in more complex systems. In the body of the paper exact expressions to thermodynamic potentials for a system of $N$ independent harmonic oscillators and the calculation of the density of states are presented; approximate expressions for the same quantities are obtained using the Mellin transform approach and the Euler-Maclaurin summation rule. Emphasis is given for the behavior of thermodynamic potentials in the higher temperature regime in order to compare the approximate expressions with the exact solution. Finally, in the Conclusions, the advantages to use the Euler-Maclaurin or Mellin transform methods is given. We present some explicit calculations necessary for a complete understanding of the paper in the appendices.

\section{Thermodynamics Potentials of System with $N-$ Independent Harmonic Oscillators}

The density of states for the $N$ independent harmonic oscillator is a basic calculation done in many textbooks (HUANG, 1987; PATHRIA, 1996) with the use of binomials coefficients. However this system is an appropriated laboratory in order get experience and to be sure about the reliability of the Mellin transform and Euler-Maclaurin methods, when applied to more complicate systems as for example in the calculation of the partition functions for the system composed by $N-$ or infinite oscillators with different frequencies, partition functions in supergravity and strings theory, and so.

The partition function is defined as (PATHRIA, 1996)

$$
Z(\beta)=\sum_{r} e^{-\beta E_{r}} ; \quad \beta \equiv \frac{1}{k_{B} T}
$$

For one simple harmonic oscillator with energy $\varepsilon_{n}=(n+$ $1 / 2) \hbar \omega$ is given by (HUANG, 1987; PATHRIA, 1996)

$$
Z_{1}(\beta)=\frac{1}{2 \sinh \left(\frac{\beta \hbar \omega}{2}\right)} .
$$

Yet, for a system of $N$ - independents, one dimensional and distinguishable harmonic oscillators we have

$$
Z_{N}(\beta)=\frac{e^{-\frac{N}{2} \hbar \omega \beta}}{\left(1-e^{-\beta \hbar \omega}\right)^{N}}=\left[2 \sinh \left(\frac{\beta \hbar \omega}{2}\right)\right]^{-N}
$$

The thermodynamic potentials: internal energy, Helmholtz free energy and entropy can be get from the partition function as

$$
\begin{aligned}
& F=-\frac{1}{\beta} \ln Z, \\
& E=F+\frac{1}{k_{B} \beta} S, \\
& S=k_{B} \beta^{2} \frac{\partial F}{\partial \beta} .
\end{aligned}
$$

By using the equation (2) in the equations (3)-(5) we get the thermodynamic potentials

$F=\frac{N}{\beta} \ln \left[2 \sinh \left(\frac{\beta \hbar \omega}{2}\right)\right]=\frac{N \hbar \omega}{2}+\frac{N}{\beta} \ln \left(1-e^{-\beta \hbar \omega}\right)$,

$$
\begin{aligned}
S & =N k_{B}\left\{\frac{\beta \hbar \omega}{2} \operatorname{coth}\left(\frac{\beta \hbar \omega}{2}\right)-\ln \left[2 \sinh \left(\frac{\beta \hbar \omega}{2}\right)\right]\right\} \\
& =\frac{N k_{B} \beta \hbar \omega}{e^{\beta \hbar \omega}-1}-N k_{B} \ln \left(1-e^{-\beta \hbar \omega}\right) \\
E & =\frac{N \hbar \omega}{2} \operatorname{coth}\left(\frac{\beta \hbar \omega}{2}\right)=\frac{N \hbar \omega}{2}+\frac{N \hbar \omega}{e^{\beta \hbar \omega}-1}
\end{aligned}
$$


It is more appropriated to rewrite these equations in terms of exponentials in order to consider the asymptotic behavior in higher temperatures,$\beta \longrightarrow 0$. As $e^{-\beta \hbar \omega} \leq 1$, it is correct to write

$$
\begin{aligned}
F & =\frac{N \hbar \omega}{2}-\frac{N}{\beta} \sum_{k=1}^{\infty} \frac{e^{-\beta \hbar \omega k}}{k}, \\
S & =N k_{B} \frac{\beta \hbar \omega}{e^{\beta \hbar \omega}-1}+N k_{B} \sum_{k=1}^{\infty} \frac{e^{-\beta \hbar \omega k}}{k}, \\
E & =\frac{N \hbar \omega}{2}+\frac{N \hbar \omega}{e^{\beta \hbar \omega}-1} .
\end{aligned}
$$

These are exact expressions. The expressions of the thermodynamic potentials for higher temperature, keeping terms of quadratic order in $\beta$, are (Appendix E)

$$
\begin{aligned}
& F \approx N\left[\frac{1}{\beta} \ln \beta \hbar \omega+\frac{\beta(\hbar \omega)^{2}}{24}\right], \\
& S \approx N k_{B}\left[1-\ln \beta \hbar \omega+\frac{(\beta \hbar \omega)^{2}}{24}\right], \\
& E \approx N\left[\frac{1}{\beta}+\frac{\beta(\hbar \omega)^{2}}{12}\right] .
\end{aligned}
$$

\section{Approximate Values of Thermodynamics Poten- tials in the Mellin Approach}

The partition function given by equation (2) is exact. Take into account our previously comment and in order to compare one different approach to calculate the partition function we use, in this section, the Melllin transform technic. By introducing the dimensionless parameter

$$
\mu \equiv \beta \hbar \omega,
$$

we write the thermodynamic potentials as

$$
\begin{aligned}
& F=-\frac{\hbar \omega}{\mu} \ln Z(\mu), \\
& E=F+\frac{\hbar \omega}{k_{B} \mu} S, \\
& S=k_{B} \frac{\mu^{2}}{\hbar \omega} \frac{\partial F}{\partial \mu} .
\end{aligned}
$$

In order to make easer the calculation of the functions $F$ and $E$, it is appropriated to define the notation

$$
X(\mu)=\ln Z_{N}(\mu)=-\frac{N \mu}{2}-N \ln \left(1-e^{-\mu}\right) .
$$

As $\exp \{-\mu\} \leq 1$, we can consider the expansion of the logarithm function in order to get

$$
X(\mu)=-\frac{N \mu}{2}+N \sum_{k=1}^{\infty} \frac{e^{-\mu k}}{k} .
$$

By using the inverse Mellin transform (GRADSHTEYN, 1984) we can write

$$
e^{-\mu k}=\frac{1}{2 \pi_{l}} \int_{c-l \infty}^{c+l \infty} \Gamma(s)(\mu k)^{-s} d s,
$$

where $c>s$. So

$-\ln \left(1-e^{-\mu}\right)=\sum_{k=1}^{\infty} \frac{e^{-\mu k}}{k}=\frac{1}{2 \pi_{l}} \int_{c-l \infty}^{c+l \infty} \frac{\Gamma(s) \zeta(s+1)}{\mu^{s}} d s$,

and

$$
X(\mu)=-\frac{N \mu}{2}+\frac{N}{2 \pi l} \int_{c-l \infty}^{c+l \infty} \frac{\Gamma(s) \zeta(s+1)}{\mu^{s}} d s .
$$

The gamma function has simple poles at $s=-n, n \in \mathbb{N}$ with residue $(-1)^{n} /(n)$ ! The function $\zeta(s+1)$ has one simple pole at $s=0$ with residue 1 . Once $\zeta(s+1)$ is finite at the point $s=-1$, the integrand has a simple pole of the gamma function; as for $s=0$ the zeta functions has a simple pole and also the gamma function, in this form the integrand has a second order pole at $s=0$. For the cases when $s=-2 n-1$, with $n \geq 1$, with the help of the reflection formula for the zeta function (BATEMAN, 1953)

$$
\zeta(s)=\frac{2 \Gamma(1-s)}{(2 \pi)^{1-s}} \operatorname{sen}\left(\frac{\pi s}{2}\right) \zeta(1-s),
$$

we can prove the analyticity of the integrand. In the points $s=-2 n$, the gamma function $\Gamma(-2 n)$, has simple poles, but $\zeta(1-2 n)$ is finite, therefore the integrand has simple poles at $s=-2 n, n \geq 1$.

All this considerations result in the simple pole of the integrand at $s=-1$ with residue $-1 \zeta(0) \mu=\mu / 2$, one second order pole at $s=0$ with residue

$$
f(\mu) \equiv \lim _{s \rightarrow 0} \frac{d}{d s}\left[(\mu)^{-s} s \Gamma(s) s \zeta(s+1)\right],
$$

where

$$
\begin{aligned}
f(\mu) & =\lim _{s \rightarrow 0}(\mu)^{-s}\left\{-\ln \mu \Gamma(s+1) s\left(\frac{1}{s}+\sum_{n=0}^{\infty} \frac{(-)^{n}}{n !} \gamma_{n} s^{n}\right)\right. \\
& +\frac{d \Gamma(s+1)}{d s} s\left(\frac{1}{s}+\sum_{n=0}^{\infty} \frac{(-)^{n}}{n !} \gamma_{n} s^{n}\right)+ \\
& \left.+\Gamma(s+1) \frac{d}{d s}\left[s\left(\frac{1}{s}+\sum_{n=0}^{\infty} \frac{(-)^{n}}{n !} \gamma_{n} s^{n}\right)\right]\right\} \\
& =-\ln \mu+\left.\Gamma(s+1) \psi(s+1)\right|_{s=0} \\
& +\left.(\mu)^{-s} \Gamma(s+1) \gamma\right|_{s=0} \\
& =-\ln \mu,
\end{aligned}
$$

(here we used the series expansion of the gamma function, $\gamma$ is the Euler-Mascheroni constant (BATEMAN, 1953) and the first order poles at $s=-2 n$, with residue

$$
\begin{array}{r}
\operatorname{Res}\left[\frac{\Gamma(s) \zeta(s+1)}{(\mu)^{s}}\right]_{s=-2 n}=\lim _{s \rightarrow-2 n}\left[\frac{(s+2 n) \Gamma(s) \zeta(s+1)}{(\mu)^{s}}\right] \\
=\frac{(\mu)^{2 n}}{(2 n) !} \zeta(1-2 n)=-\frac{(\mu)^{2 n} B_{2 n}}{2 n(2 n) !},
\end{array}
$$


where $B_{n}$ are the Bernoulli numbers, Appendix C.

By using the previous results in the equation (16) we have

$$
\begin{aligned}
X(\mu) & =-\frac{N \mu}{2}+N\left[\left(\frac{\mu}{2}-\ln \mu\right)+\sum_{n=1}^{\infty} \frac{\mu^{2 n}}{(2 n) !} \zeta(1-2 n)\right] \\
& =-N \ln \mu+N \sum_{n=1}^{\infty} \frac{\mu^{2 n}}{(2 n) !} \zeta(1-2 n) .
\end{aligned}
$$

These equations are exacts. The thermodynamic potentials, equations (13)-(15), in terms of the function $X(\mu)$ are

$$
\begin{aligned}
& F=\frac{N \hbar \omega}{\mu}\left[\ln \mu-\sum_{n=1}^{\infty} \frac{\mu^{2 n}}{(2 n) !} \zeta(1-2 n)\right], \\
& S=k_{B} N\left[1-\ln \mu-\sum_{n=1}^{\infty} \frac{(2 n-1) \mu^{2 n}}{(2 n) !} \zeta(1-2 n)\right], \\
& E=\frac{N \hbar \omega}{\mu}\left[1-\sum_{n=1}^{\infty} \frac{\mu^{2 n}}{(2 n-1) !} \zeta(1-2 n)\right] .
\end{aligned}
$$

These expressions can be rewrite whit the help of the equation (12) and $\beta$ as

$F=\frac{N}{\beta}\left[\ln \beta \hbar \omega-\sum_{n=1}^{\infty} \frac{(\beta \hbar \omega)^{2 n}}{(2 n) !} \zeta(1-2 n)\right]$

$S=k_{B} N\left[1-\ln \beta \hbar \omega-\sum_{n=1}^{\infty} \frac{(2 n-1)(\beta \hbar \omega)^{2 n}}{(2 n) !} \zeta(1-2 n)\right]$,

$E=\frac{N}{\beta}\left[1-\sum_{n=1}^{\infty} \frac{(\beta \hbar \omega)^{2 n}}{(2 n-1) !} \zeta(1-2 n)\right]$.

The main contribution, of $\beta^{2}$ order, is easily to get from this equations as, (Appendix B):

$$
\begin{aligned}
F & \approx N\left[\frac{1}{\beta} \ln \beta \hbar \omega+\frac{\beta}{24}(\hbar \omega)^{2}\right], \\
S & \approx k_{B} N\left[1-\ln \beta \hbar \omega+\frac{(\beta \hbar \omega)^{2}}{24}\right], \\
E & \approx N\left[\frac{1}{\beta}+\frac{\beta(\hbar \omega)^{2}}{12}\right] .
\end{aligned}
$$

At this point we can compare the equations (9)-(11) with equations (19)-(21). The first ones was got as approximations from the exact results for the partition function. The second set of equations was obtained by using the Mellin transform (residue calculation) in order to calculate the sum over permissible value of the energy of the system. This procedure provide as a results, the power series representation for the partition function, and as a consequence the power series representation for the thermodynamic potentials.

The comparison of these equation show us the perfect agreement for the results obtained using the Mellin transform technic, in the second order approximation in $\beta$, with the series representation, equations (9)-(11), of the exact equations for the thermodynamic potentials equations (6) and (7). Taking as example, the exact equivalence of the expression for the Helmoltz free energy in the Mellin approach and the exact one expression is presented in Appendix F.

\section{The Euler-Maclaurin Summation Rules Applied to Calculation of Approximate Values of the Thermodynamic Potential}

Let

$$
\ln Z_{N}(\mu)=Y(\mu)=-\frac{N \mu}{2}+N \sum_{k=1}^{\infty} \frac{e^{-\mu k}}{k} .
$$

By using the Euler-Maclaurin summation formula (ABRAMOWITZ; STEGUN, 1964), (Appendix D) we have

$$
\begin{aligned}
\frac{Y(\mu)}{N} & =-\frac{\mu}{2}+\frac{e^{-\mu}}{2}+\Gamma[0, \mu]+\frac{1}{12} e^{-\mu}(1+\mu) \\
& +\sum_{k=2}^{\infty} \frac{B_{2 k}}{(2 k) !}\left[f^{(2 k-1)}(n)-f^{(2 k-1)}(0)\right],
\end{aligned}
$$

where

$$
\Gamma[a, x]=\int_{x}^{\infty} t^{a-1} e^{-t} d t
$$

is the incomplete gamma function.

The thermodynamic potentials, equations (13)-(15), in terms of $Y(\mu)$ are

$$
\begin{aligned}
& F= \frac{N \hbar \omega}{\mu}\left\{\frac{\mu}{2}-\frac{e^{-\mu}}{2}-\Gamma[0, \mu]-\frac{1}{12} e^{-\mu}(1+\mu)\right. \\
&\left.-\sum_{k=2}^{\infty} \frac{B_{2 k}}{(2 k) !}\left[\left.f^{(2 k-1)}(k)\right|_{k \rightarrow \infty}-f^{(2 k-1)}(0)\right]\right\}, \\
& S= \frac{N k_{B}}{12} e^{-\mu}\left[19+\mu(7+\mu)+12 e^{\mu} \Gamma[0, \mu]\right] \\
&-N k_{B} \mu^{2} \frac{\partial}{\partial \mu}\left\{\frac{1}{\mu} \sum_{k=2}^{\infty} \frac{B_{2 k}}{(2 k) !}\left[\left.f^{(2 k-1)}(k)\right|_{k \rightarrow \infty}-f^{(2 k-1)}(0)\right]\right\}, \\
& E=\frac{N \hbar \omega}{\mu}\left[\frac{\mu}{2}+e^{-\mu}\left(1+\frac{\mu}{2}+\frac{\mu^{2}}{12}\right)\right] \\
&-N k_{B} \mu \frac{\partial}{\partial \mu} \sum_{k=2}^{\infty} \frac{B_{2 k}}{(2 k) !}\left[f^{(2 k-1)}(k)_{k \rightarrow \infty} \mid-f^{(2 k-1)}(0)\right] .
\end{aligned}
$$

From these equations, in the order of $\beta^{2}$, Appendix G, we have

$$
\begin{aligned}
F & \approx N\left[\frac{1}{\beta} \ln \beta \hbar \omega+\frac{\beta}{24}(\hbar \omega)^{2}\right], \\
S & \approx N k_{B}\left[1-\ln \beta \hbar \omega+\frac{(\beta \hbar \omega)^{2}}{24}\right], \\
E & \approx N\left[\frac{1}{\beta}+\frac{\mu(\hbar \omega)^{2}}{12}\right] .
\end{aligned}
$$

As we can see these expressions agree with previous ones, equations (19)-(21). 


\section{Comments and Conclusion}

The goal of this paper is to compare two technics: Mellin transform and Euler-Maclaurin summation formula in order do decide what is the more appropriated, between these, to calculate finite and infinite characteristics sums of the thermodynamical systems order to get insight and experience to consider more involved problems like blackhole entropy, partitions functions of gas in the presence of gravitational fields, etc.

To carry out this calculation, we consider one very well know and exactly soluble systems composite of $N$ identical simple harmonic oscillators, with energy eigenvalue $(n+1 / 2) \hbar \omega$, in order to exactly calculated the thermodynamic potentials $F(\beta, N), S(\beta, N)$ e $E(\beta, N)$. In the sequence, we rewrite these potentials in $\beta$ power series, equations (9)-(11), in order to study the mains contributions at higher temperature, $\beta \rightarrow 0$, at list, until the $\beta^{2}$ order. The logarithm divergent term, typical of this kinds of models, that immediately appear in this expansion, are associated with origin divergence.

In this paper, the Mellin transform and the EulerMaclaurin summation formula was used in order to calculate the approximate expression of the finite sum, the partition function $Z(\beta)$, and as consequence, approximate expressions for the thermodynamic potentials that, also, was expressed as power series in the temperature $\beta$ as shown in the equations (19)-(21).

In technical, not physical, point of view, from comparison of equations (9)-(11) with the equations (19)-(21) is clear the agreement of this equations, even form small numbers of terms in the expansion in power series of $\beta$. It is a good result, one times we deal with more complicated systems, we can be sure that the method give quite good estimations for the calculation of the physical quantities even for small number of terms, for the both methods. Also, from the calculation, we conclude that the Mellin transform methods is more simple to apply in comparison with the Euler-Maclaurin one. The reason is the divergences in the calculation of the $(2 k-1)$ th derivatives of the function $f(k)$ in the origin, in the Euler-Maclaurin summation formula, equation (30). It is worthy to point that we haven't take into account the thirty order derivative of the function $f(k)$ since the non null contribution of this term beginning at 4th order. However, in the EulerMaclaurin method, even for second order contribution there is an interesting result. In the approximate expression for the entropy $S$, given by equation (10), calculated from the exact partition function $Z(\beta)$, equation (2) there is the contribution

$$
1-\ln \mu,
$$

but in the calculation of the approximate expression for entropy, carried out in the Appendix G was got the contribution $\frac{19}{12}-\gamma$ in the expression

$$
N k\left[\frac{19}{12}-\gamma-\ln \mu+\frac{\mu^{2}}{24}\right] .
$$

A simple calculation show us that

$$
\frac{19}{12}-\gamma=1.583333-0.577215=1.00611,
$$

meaning that even for expansion until second order in the calculation of the entropy, with Euler-Maclaurin summation formula, this result differs only by $0.6 \%$ of the exact value 1 . However it should not that the Mellin transform methods immediately gives the exact value 1 .

\section{Acknowledgement}

The authors wold like to acknowledge the PROPPG/UEL by support in the project $\mathrm{n}^{\circ} 09798$.

\section{Appendix A: The Mellin Transform}

The Mellin transform of the function $f(x)$ (GRADSHTEYN, 1984) denoted by $f^{*}(s)$, is defined by the integral

$$
f^{*}(s)=\int_{0}^{\infty} f(x) x^{s-1}, \quad s \in \mathbb{C} .
$$

The functions $f(x)$ and $f^{*}(s)$ are called a Mellin transform pair, and knowledge of either one enables the other to be recovered. The transform exists, provide the integral

$$
\int_{0}^{\infty}|f(x)| x^{k-1} d x
$$

is bounded for some $k>0$.

The inverse Mellin transform is the integral

$$
f(x)=\frac{1}{2 \pi_{l}} \int_{c-l \infty}^{c+l \infty} f^{*}(s) x^{s-1} d s, \quad c>k .
$$

The standard notation

$$
f^{*}(s)=\mathscr{M}[f(x) ; s],
$$

is used to represent the Mellin transformation.

\section{Appendix B: The Riemann Zeta, and Related Functions}

The Riemann zeta function, firstly introduced by Euler for real $s>1$ as the series

$$
1+\frac{1}{2^{s}}+\frac{1}{3^{s}}+\frac{1}{4^{s}} \cdots,
$$


is defined as

$$
\zeta(s)=\sum_{n=1}^{\infty} \frac{1}{n^{s}}, s \in \mathbb{C}, \quad \Re(s)>1 .
$$

Riemann had extended the definition of the zeta function for complex number $s$ by analytic extension of the Euler representation, equation (26). There are many ways to analytically extended this function, all of them valid as the results is independent of the technic by taking into account the unicity of methods. There is too the so called Dirichlet eta function, associated to infinite alternating series,

$$
\eta(s)=\sum_{n=1}^{\infty} \frac{(-)^{n-1}}{n^{s}},
$$

analytic for $\Re(s)>0$. If the complex number $s$ satisfy $\mathfrak{R}(s)>1$, we have

$$
\begin{aligned}
\zeta(s)-\eta(s) & =\sum_{n=1}^{\infty} \frac{1}{n^{s}}-\sum_{n=1}^{\infty} \frac{(-)^{n-1}}{n^{s}}=\sum_{n=1}^{\infty}\left(\frac{1}{n^{s}}+\frac{(-)^{n}}{n^{s}}\right) \\
& =\left(\frac{1}{1^{s}}-\frac{1}{1^{s}}\right)+\left(\frac{1}{2^{s}}+\frac{1}{2^{s}}\right) \\
& +\left(\frac{1}{3^{s}}-\frac{1}{3^{s}}\right)+\cdots \\
& =2 \frac{1}{2^{s}}+2 \frac{1}{4^{s}}+2 \frac{1}{6^{s}}+\cdots \\
& =2\left(\frac{1}{2^{s}}+\frac{1}{4^{s}}+\frac{1}{6^{s}}+\cdots\right) \\
& =2 \sum_{n=1}^{\infty} \frac{1}{(2 n)^{s}} .
\end{aligned}
$$

This expression can be rewrite as

$$
\begin{aligned}
\zeta(s)-\eta(s) & =\frac{2}{2^{s}} \sum_{n=1}^{\infty} \frac{1}{n^{s}}=2^{1-s} \zeta(s) \Longrightarrow \\
\eta(s) & =\left(1-2^{1-s}\right) \zeta(s) .
\end{aligned}
$$

This is a basic and very useful equation relating the both, the zeta and eta functions. The Riemann zeta function in the integral representation is

$$
\zeta(s)=\frac{1}{\Gamma(s)} \int_{0}^{\infty} d t \frac{t^{s-1}}{e^{t}-1} .
$$

The integral representation shows that $\zeta(s)$ can be analytically continued and that is one-value everywhere except for $s=1$ where it has a single pole with residue 1 . One useful representation of the zeta function, obtained from Laurent series, is

$$
\zeta(s)=\frac{1}{s-1}+\gamma+\sum_{n=1}^{\infty} \gamma_{n}(s-1)^{n}
$$

where

$$
\gamma_{n}=\lim _{m \rightarrow \infty}\left[\sum_{l=1}^{m} \frac{(\ln l)^{n}}{l}-\frac{(\ln m)^{n+1}}{(n+1)}\right],
$$

are the so called Stieltjes constants.
Some useful values of the Riemann zeta function (GRADSHTEYN, 1984)

$$
\begin{aligned}
& \zeta(2 m)=\frac{2^{m-1} \pi^{2 m}\left|B_{2 m}\right|}{(2 m) !}, \quad \zeta(1-2 m)=-\frac{B_{2 m}}{2 m}, \\
& \zeta(-2 m)=0, \\
& \left.\frac{d \zeta(s)}{d s}\right|_{s=0}=-\frac{1}{2} \ln 2 \pi, \quad \zeta(-1)=-\frac{1}{12}, \\
& \zeta(0)=-\frac{1}{2}, \quad \zeta(1)=\infty .
\end{aligned}
$$

\section{Appendix C: The Bernoulli Numbers}

The Bernoulli numbers, $B_{n}$, are a sequence of signed rational numbers that can be defined by the generating function (ABRAMOWITZ; STEGUN, 1964; GRADSHTEYN, 1984)

$$
\frac{t}{e^{t}-1}=\sum_{n=0}^{\infty} B_{n} \frac{t^{n}}{n !}, 0<|t|<2 \pi,
$$

as the coefficients in the power series of $t^{n} / n$ !.

Some useful values of Bernoulli numbers (GRADSHTEYN, 1984)

$$
\begin{array}{cl}
B_{0}=1, & B_{1}=-\frac{1}{2}, \quad B_{2}=-\frac{1}{6}, \quad B_{1}=-\frac{1}{30} \\
& B_{2 n+1}=0, \quad n=1,2, \cdots
\end{array}
$$

\section{Appendix D: The Euler-Maclaurin Summation Formula}

The Euler-Maclaurin summation formula has several representations, one of which is (ABRAMOWITZ; STEGUN, 1964)

$$
\begin{aligned}
\sum_{k=1}^{n-1} f_{k} & =\int_{0}^{n} f(k) d k-\frac{1}{2}[f(0)+f(n)] \\
& +\frac{1}{12}\left[f^{\prime}(n)-f^{\prime}(0)\right]-\frac{1}{720}\left[f^{\prime \prime \prime}(n)-f^{\prime \prime \prime}(0)\right] \\
& +\frac{1}{30240}\left[f^{V}(n)-f^{V}(0)\right]+\cdots
\end{aligned}
$$

or in terms of Bernoulli numbers as

$$
\begin{aligned}
\sum_{k=1}^{n-1} f_{k} & =\int_{0}^{n} f(k) d k-\frac{1}{2}[f(0)+f(n)] \\
& +\frac{1}{12}\left[f^{\prime}(n)-f^{\prime}(0)\right] \\
& +\sum_{k=1}^{\infty} \frac{B_{2 k}}{(2 k) !}\left[f^{(2 k-1)}(n)-f^{(2 k-1)}(0)\right] .
\end{aligned}
$$


Appendix E: Detailed Calculation of the Thermodynamic Potentials in the Quadratic Approximation

The free energy power series in $\beta$ can be get from the series representation of the hyrpebolic sin or from the exponential representation, what have some subtlety. Of course both way give from the equation (3) the same expression for the free energy

$$
\begin{gathered}
F=\frac{N \hbar \omega}{2}+\frac{N}{\beta} \ln \left(1-e^{-\beta \hbar \omega}\right) \approx \frac{N \hbar \omega}{2}+\frac{N}{\beta} \ln \beta \hbar \omega \\
-\frac{N \hbar \omega}{2}+\frac{N \beta(\hbar \omega)^{2}}{24}=\frac{N}{\beta} \ln \beta \hbar \omega+\frac{N \beta(\hbar \omega)^{2}}{24} .
\end{gathered}
$$

For the entropy, $S$, we have from the equation (5)

$$
\begin{aligned}
S & =N k_{B} \frac{\beta \hbar \omega}{e^{\beta \hbar \omega}-1}+N k_{B} \sum_{k=1}^{\infty} \frac{e^{-\beta \hbar \omega k}}{k} \\
\approx N k_{B} & {\left[1-\frac{\mu}{2}+\frac{\mu^{2}}{12}-\ln \mu+\frac{\mu}{2}-\frac{(\mu)^{2}}{24}\right] } \\
& =N k_{B}\left[1+\frac{\mu^{2}}{24}-\ln \mu\right] .
\end{aligned}
$$

The internal energy of the system can easer be get from the equation (8), that have the form of the generating function of the Bernoulli numbers (GRADSHTEYN, 1984)

$$
\begin{aligned}
E & =\frac{N \hbar \omega}{2}+\frac{N \hbar \omega}{e^{\beta \hbar \omega}-1}=N \hbar \omega\left[\frac{1}{2}+\left(\frac{1}{\mu}-\frac{1}{2}+\frac{\mu}{12}\right)\right] \\
& +O\left(\mu^{3}\right) \approx N \hbar \omega\left[\frac{1}{\mu}+\frac{\mu}{12}\right] .
\end{aligned}
$$

\section{Appendix F: Equivalence Between the Expres-} sions for the Helmholtz Free Energy in Different Approaches

The second term in the free energy, equation (6),

$$
F=\frac{N \hbar \omega}{2}+\frac{N}{\beta} \ln \left(1-e^{-\mu}\right),
$$

can be write as

$\frac{N}{\beta} \ln \left(1-e^{-\mu}\right)=\frac{N}{\beta}\left[\ln \left(1+e^{-\mu / 2}\right)+\ln \left(1-e^{-\mu / 2}\right)\right]$.

Let as consider the function $\ln \left(1+e^{-\mu / 2}\right)$. As $e^{-\mu} \leq 1$, and by define

$$
\alpha \equiv \mu / 2
$$

it is correct to write

$$
\ln \left(1+e^{-\alpha}\right)=e^{-\alpha}-\frac{e^{-2 \alpha}}{2}+\frac{e^{-3 \alpha}}{3}-\frac{e^{-4 \alpha}}{4}+\cdots .
$$

By expanding each one of the exponentials in the power series, we have

$$
\begin{aligned}
\ln \left(1+e^{-\alpha}\right)= & \left(\frac{1}{1}\right)\left[1-\alpha+\frac{\alpha^{2}}{2 !}-\frac{\alpha^{3}}{3 !}+\cdots\right] \\
& -\left(\frac{1}{2}\right)\left[1-2 \alpha+\frac{4 \alpha^{2}}{2 !}-\frac{8 \alpha^{3}}{3 !}+\cdots\right] \\
& +\left(\frac{1}{3}\right)\left[1-3 \alpha+\frac{9 \alpha^{2}}{2 !}-\frac{27 \alpha^{3}}{3 !}+\cdots\right] \\
& -\cdots
\end{aligned}
$$

Collecting the commons terms in the $\alpha$ power, we have

$$
\begin{aligned}
\ln \left(1+e^{-\mu / 2}\right)= & \underbrace{1-\frac{1}{2}+\frac{1}{3}-\frac{1}{4}+\cdots}_{\eta(1)} \\
& -\alpha(\underbrace{1+1-1+1-\cdots}_{\eta(0)}) \\
& +\frac{(\alpha)^{2}}{2 !}(\underbrace{1-2+3-4+\cdots}_{\eta(-1)}) \cdots \\
& =\eta(1)-\alpha \eta(0)+\frac{(\alpha)^{2}}{2 !} \eta(-1)+\cdots
\end{aligned}
$$

Now, consider the function $\ln \left(1-e^{-\mu / 2}\right)$ in the equation (31); we also can write

$$
\begin{aligned}
\ln \left(1-e^{-\alpha}\right)= & \ln \left[\left(1+e^{-\alpha \mathrm{t} / 2}\right)\left(1+e^{-\alpha / 4}\right)\right. \\
& \left.\left(1-e^{-\alpha / 4}\right)\right] \\
= & \ln \left(1+e^{-\alpha / 2}\right)+\ln \left(1+e^{-\alpha / 4}\right) \\
+ & \ln \left(1-e^{-\alpha / 4}\right),
\end{aligned}
$$

and, by continue this process $N$ times, we have

$$
\begin{aligned}
f(\alpha) \equiv \ln \left(1-e^{-\alpha}\right)= & \sum_{n=1}^{N} \ln \left(1+e^{-\alpha / 2^{n}}\right) \\
& +\ln \left(1-e^{-\alpha / 2^{N}}\right) .
\end{aligned}
$$

We can substitute for each term in the sum of the equation (36) the expansion, equation (34), taking into account that 
each term of this sum contributes with $\mu / 2^{N}$, then

$$
\begin{aligned}
f(\alpha)= & \sum_{n=1}^{N}\left[\eta(1)-\frac{\alpha}{2^{n}} \eta(0)\right. \\
& \left.+\frac{1}{2 !}\left(\frac{\alpha}{2^{n}}\right)^{2} \eta(-1)+\cdots\right] \\
& +\ln \left(1-e^{-\alpha / 2^{N}}\right) \\
& =\eta(1) N-\eta(0)(\alpha) \frac{1}{2} \sum_{n=1}^{N} \frac{1}{2^{n-1}} \\
& +\frac{(\alpha)^{2}}{2 !} \eta(-1) \frac{1}{2^{3}} \sum_{n=1}^{N} \frac{1}{2^{2 n-1}} \\
& -\frac{(\alpha)^{3}}{3 !} \eta(-2) \frac{1}{2^{4}} \sum_{n=1}^{N} \frac{1}{2^{3 n-1}}+\cdots \\
& +\ln \left(1-e^{-\alpha / 2^{N}}\right)
\end{aligned}
$$

By taking the limit where $N$ goes to infinity and evaluating each geometric series we have

$$
\begin{aligned}
f(\alpha) & =\lim _{N \rightarrow \infty} N \eta(1)-\eta(0)(\alpha) \frac{1}{2} 2 \\
& +\frac{(\alpha)^{2}}{2 !} \eta(-1) \frac{1}{2^{3}} \frac{2}{3} \\
& -\frac{(\alpha)^{3}}{3 !} \eta(-2) \frac{1}{2^{4}} \frac{2}{7} \cdots \\
& +\ln \left(1-e^{-\alpha / 2^{N}}\right)
\end{aligned}
$$

By using this results, the values of $\eta(-2)=0, \eta(-1)=$ $1 / 4, \eta(0)=1 / 2, \eta(1)=\ln 2, \ldots$, the equation (38) can be rewrite as

$$
\begin{aligned}
f(\alpha) & =\lim _{N \rightarrow \infty} N \ln 2-\frac{1}{2}(\alpha) \\
& +\frac{(\alpha)^{2}}{2 !} \frac{1}{2^{4}} \frac{1}{3}-0+\cdots \\
& +\ln \left(1-e^{-\alpha / 2^{N}}\right)
\end{aligned}
$$

For large value of $N$ we have the approximation

$$
1-e^{-\alpha / 2^{N}} \approx \frac{\alpha}{2^{N}}
$$

and therefore imply that

$$
\ln \left(1-e^{-\alpha / 2^{N}}\right) \approx \ln \alpha-N \ln 2 .
$$

By using this results in the equation (39) we have

$$
f(\alpha)=\ln \alpha-\frac{\alpha}{2}+\frac{\alpha^{2}}{2 !} \frac{1}{2^{4}} \frac{1}{3}-0+\cdots .
$$

With the help of the equations (31), (34), (40), the equation (6) can be rewrite as

$$
\begin{aligned}
F= & \frac{N \hbar \omega}{2}+\frac{N}{\beta}\left[\eta(1)-\alpha \eta(0)+\frac{(\alpha)^{2}}{2 !} \eta(-1)+\cdots\right. \\
& \left.+\ln \alpha-\frac{\alpha}{2}+\frac{\alpha^{2}}{2 !} \frac{1}{2^{4}} \frac{1}{3}-0+\cdots\right] .
\end{aligned}
$$

By using the relation between the eta and zeta functions, $\eta(s)=\left(1-2^{1-s}\right) \zeta(s)$, and the equations (12),(32), the expression of the Helmholtz fee energy, equation (41), result totally equivalent to the expansion obtained by using the Mellin transform approach, equation (18),

$$
F=\frac{N \hbar \omega}{\mu}\left[\ln \mu-\sum_{n=1}^{\infty} \frac{\mu^{2 n}}{(2 n) !} \zeta(1-2 n)\right]
$$

\section{Appendix G: The Approximated Expressions for Thermodynamic Potentials in the Euler- Maclaurin Approach}

The Free energy $F$ in the approximation $\mu^{2}$ without the $B_{4}$ contribution

$$
\begin{aligned}
F & \approx N \hbar \omega\left[\frac{1}{2}-\frac{e^{-\mu}}{2 \mu}-\frac{\Gamma[0, \mu]}{\mu}-\frac{1}{12} \frac{e^{-\mu}}{\mu}(1+\mu)\right] \\
& =N \hbar \omega\left[\frac{1}{2}-\left(\frac{1}{2 \mu}-\frac{1}{2}+\frac{\mu^{4}}{4}-\frac{\mu^{2}}{12}\right)\right. \\
& +\left(\frac{\gamma}{\mu}+\frac{1}{\mu} \ln \mu-\AA^{4}+\frac{\mu^{4}}{4}-\frac{\mu^{2}}{18}\right)-\frac{1}{12}\left(\frac{1}{\mu}-\frac{\mu}{2}+\frac{\mu^{2}}{3}\right) \\
& \approx \frac{N}{\beta} \ln \beta \hbar \omega+\frac{N \beta}{24}(\hbar \omega)^{2} .
\end{aligned}
$$

Exact Entropy $S$

$$
\begin{aligned}
S & =k_{B} \frac{\mu^{2}}{\hbar \omega} \frac{\partial F}{\partial \mu} \\
& =\frac{N k_{B}}{12} e^{-\mu}\left[19+\mu(7+\mu)+12 e^{\mu} \Gamma[0, \mu]\right] \\
& -k_{B} \mu^{2}\left\{\frac{\partial}{\partial \mu} \frac{1}{\mu} \sum_{k=2}^{\infty} \frac{B_{2 k}}{(2 k) !}\left[f^{(2 k-1)}(n)-f^{(2 k-1)}(0)\right]\right\} .
\end{aligned}
$$

Approximated Entropy $S$, with contribution of $(1 / 12)\left[f^{\prime}(n)-f^{\prime}(0)\right]$

$$
\begin{aligned}
S & =\frac{N k_{B}}{12} e^{-\mu}\left[19+\mu(7+\mu)+12 e^{\mu} \Gamma[0, \mu]\right]+O\left(B_{4}\right) \\
& \approx \frac{N k_{B}}{12}\left(19-12 \mu+\frac{7 \mu^{2}}{2}\right)+N k_{B}\left(-\gamma-\ln \mu+\mu-\frac{\mu^{2}}{4}\right) \\
& \approx N k_{B}\left[1-\ln \beta \hbar \omega+\frac{(\beta \hbar \omega)^{2}}{24}\right] .
\end{aligned}
$$




\section{References}

ABRAMOWITZ, M.; STEGUN, I. A., Handbook of mathematical functions: with formulas, graphs, and mathematical tables. [S.1.]: Courier Corporation, 1964. v. 55.

BATEMAN, H., Higher Transcendental Functions. [S.1.]: Mack-Graw-Hill Book Company Inc., New York, USA, 1953. v. 1.

BOHR, N.; KALCKAR, F., On the transmutation of atomic nuclei by impact of material particles. Matematiskfysiske Meddelelser udfivet af Det Kongelige Danske Videnskabernes Selskab, v. 14, n. 10, p. 1-40, 1937.

GRADSHTEYN, I. M. R. I. S., Tables of Integrals, Series, and Products. [S.1.]: Academic Press Inc., New York, 1984.

HUANG, K., Statistical Mechanics. Second. [S.1.]: John Wiley \& Sons Inc., New York, USA, 1987.

PATHRIA, P. D. B. R. K., Statistical mechanics. 2nd ed. ed. [S.1.]: Butterworth-Heinemann, 1996. ISBN $0750624698,9780750624695,9780080541716$.

POLCHINSKI, J., String theory: Volume 1, superstring theory and beyond. [S.1.]: Cambridge university press, 1998.

POLCHINSKI, J., String theory: Volume 2, superstring theory and beyond. [S.1.]: Cambridge university press, 1998.

STROMINGER, A.; VAFA, C., Microscopic origin of the bekenstein-hawking entropy. Physics Letters B, Elsevier, v. 379, n. 1-4, p. 99-104, 1996.

WITTEN, E., Topological Gravity. Physics Letters, B206, p. 601-606, 1988.

WITTEN, E., Topology Changing Amplitudes in (2+1)Dimensional Gravity. Nuclear Physics, B323, p. 113-140, 1989.

WITTEN, E., On the Structure of the Topological Phase of Two-dimensional Gravity. Nuclear Physics, B340, p. 281-332, 1990.

WITTEN, E., Quantum gravity in de Sitter space. In: Strings 2001: International Conference Mumbai, India, January 5-10, 2001. [ s.n.], 2001.

WITTEN, E., Gauge theory and gravity. Conference Proceedings, C0208124, p. 8-13, 2002.
WITTEN, E., Three-dimensional gravity revisited. arXiv preprint arXiv:0706.3359, 2007. 